\title{
OCTP: A Tool for On-the-Fly Calculation of Transport Properties of Fluids with the Order-n Algorithm in LAMMPS
}

\section{Journal Article}

\section{Author(s):}

Jamali, Seyed H.; Wolff, Ludger; Becker, Tim M.; de Groen, Mariëtte; Ramdin, Mahinder; Hartkamp, Remco; Bardow, André (iD; Vlugt, Thijs J.H.; Moultos, Othonas A.

\section{Publication date:}

2019-04-22

\section{Permanent link:}

https://doi.org/10.3929/ethz-b-000427041

\section{Rights / license:}

Creative Commons Attribution-NonCommercial-NoDerivatives 4.0 International

\section{Originally published in:}

Journal of Chemical Information and Modeling 59(4), https://doi.org/10.1021/acs.jcim.8b00939 


\section{OCTP: A Tool for On-the-Fly Calculation of Transport Properties of Fluids with the Order- $n$ Algorithm in LAMMPS}

Seyed Hossein Jamali, ${ }^{\dagger}$ Ludger Wolff, ${ }^{\ddagger}$ Tim M. Becker, ${ }^{\dagger}{ }^{\dagger}$ Mariëtte de Groen, ${ }^{\dagger}$ Mahinder Ramdin, ${ }^{\dagger}$ Remco Hartkamp, ${ }^{\dagger[0}$ André Bardow, ${ }^{\ddagger 0}$ Thijs J. H. Vlugt, ${ }^{\dagger}$ and Othonas A. Moultos ${ }^{*},^{\dagger}$

${ }^{\dagger}$ Engineering Thermodynamics, Process \& Energy Department, Faculty of Mechanical, Maritime and Materials Engineering, Delft University of Technology, Leeghwaterstraat 39, 2628CB Delft, The Netherlands

${ }^{\ddagger}$ Institute of Technical Thermodynamics, RWTH Aachen University, 52056 Aachen, Germany

Supporting Information

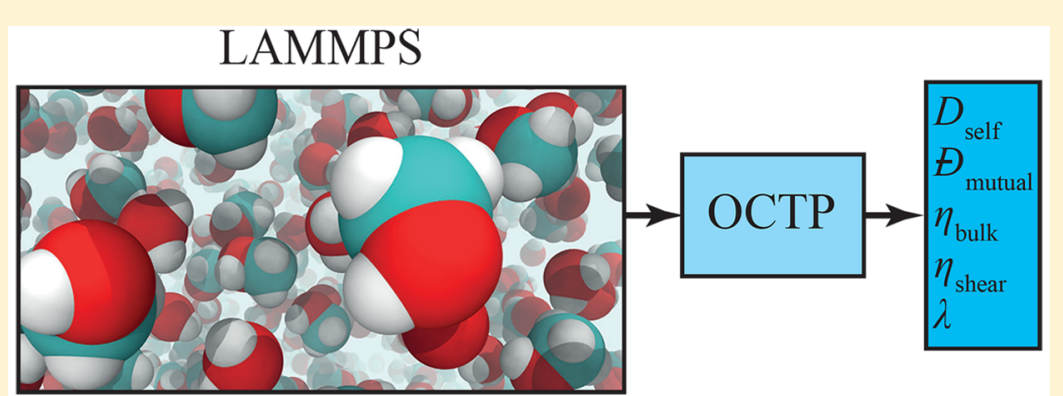

ABSTRACT: We present a new plugin for LAMMPS for on-the-fly computation of transport properties (OCTP) in equilibrium molecular dynamics. OCTP computes the self- and Maxwell-Stefan diffusivities, bulk and shear viscosities, and thermal conductivities of pure fluids and mixtures in a single simulation. OCTP is the first implementation in LAMMPS that uses the Einstein relations combined with the order- $n$ algorithm for the efficient sampling of dynamic variables. OCTP has low computational requirements and is easy to use because it follows the native input file format of LAMMPS. A tool for calculating the radial distribution function (RDF) of the fluid beyond the cutoff radius, while taking into account the system size effects, is also part of the new plugin. The RDFs computed from OCTP are needed to obtain the thermodynamic factor, which relates Maxwell-Stefan and Fick diffusivities. To demonstrate the efficiency of the new plugin, the transport properties of an equimolar mixture of water-methanol were computed at $298 \mathrm{~K}$ and 1 bar.

\section{INTRODUCTION}

Transport properties of liquids, e.g., diffusivity, viscosity, and thermal conductivity, play an important role in many environmental and industrial applications. ${ }^{1-3}$ Traditionally, transport properties are measured in experiments. ${ }^{1}$ However, such measurements are often costly and difficult to perform, especially at elevated temperatures and pressures, or when dangerous chemicals are involved. Molecular dynamics (MD) simulation is a powerful alternative for obtaining transport coefficients. ${ }^{4}$ The exponential increase in computational power in the past few decades, along with the development of robust open-source packages, such as LAMMPS, ${ }^{5}$ GROMACS, ${ }^{6}$ and $\mathrm{NAMD},{ }^{7}$ allows for the efficient use of $\mathrm{MD}$ as a part of the design and optimization of various industrial processes (Figure 1). Typical examples are gas treatment, ${ }^{8,9}$ carbon capture and sequestration, ${ }^{10-13}$ and desalination using nanoporous membranes. $^{14,15}$ Since there is a continuous demand for the prediction of transport coefficients, computational tools that are both accurate and easy-to-use are urgently needed. ${ }^{16,17}$

In the MD framework, transport properties can be obtained from either nonequilibrium (NEMD) or equilibrium (EMD) simulations. In NEMD, the response of the system to external

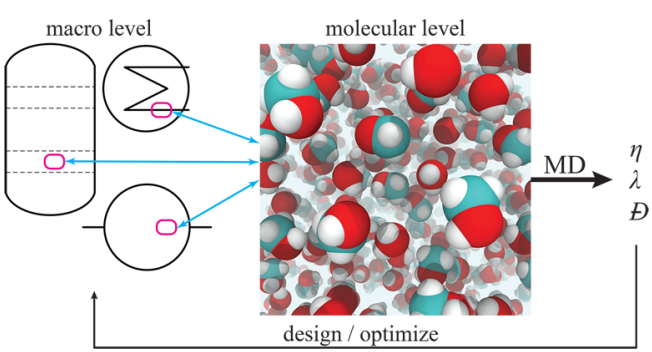

Figure 1. MD simulations can be used to compute viscosities $(\eta)$, thermal conductivities $(\lambda)$, and diffusivities $(Đ)$ at different temperature and pressure conditions. These computations can be used for the design and optimization of industrial applications.

flows or driving forces yields the transport properties. ${ }^{4}$ Properties computed from NEMD may strongly depend on the applied driving force, and thus, EMD is usually preferred. ${ }^{18}$ In EMD a transport coefficient $(\gamma)$ is related to an integral over a time-correlation function of a dynamical variable $\dot{A}: 4,19,20$

Received: December 20, 2018

Published: February 11, 2019 


$$
\gamma=\int_{0}^{\infty}\left\langle\dot{A}\left(t^{\prime}\right) \dot{A}(0)\right\rangle \mathrm{d} t^{\prime}
$$

where the angle brackets $\langle\cdots\rangle$ denote an ensemble average. Equation 1 is called the Green-Kubo relation. ${ }^{20}$ The corresponding variables $\dot{A}$ for the diffusion coefficient, viscosity, and thermal conductivity are the velocities of molecules, the components of the stress tensor, and the components of the energy current (heat flux), respectively. ${ }^{20}$ An equivalent method for obtaining transport coefficients from EMD is the Einstein method: ${ }^{4}$

$$
\gamma=\left\langle(A(t)-A(0))^{2}\right\rangle / 2 t
$$

where at large $t$, the linear relation between time $(t)$ and the mean-squared displacement (MSD) of the dynamical variable $(A(t)-A(0))^{2}$ yields the corresponding transport coefficient. ${ }^{4}$ The linear relation of eq 2 is valid at time scales where the slope of MSD as a function of time is 1 in a log-log plot., ${ }^{4,21}$ This criterion is often used to specify the minimum length of the simulation for obtaining transport properties with the Einstein method. Such a criterion is missing from the conventional Green-Kubo method, where the tail of the time-correlation functions very slowly converges toward zero regardless of the simulation length. ${ }^{22,23}$ While this issue has recently been addressed by the time-decomposition method proposed by Maginn and co-workers, ${ }^{24,25}$ from a practical point of view, the Einstein method has a clear advantage over the conventional Green-Kubo method.

The conventional method for calculating the ensemble averages in eqs 1 and 2 uses a fixed sampling frequency., ${ }^{4,21}$ This is not efficient for systems with a broad range of dynamic time scales. ${ }^{26}$ Low-frequency sampling may result in missing fast-decaying correlation functions, while high-frequency sampling requires a huge amount of computational resources for storing and postprocessing large data files. ${ }^{27}$ The efficiency of sampling can be improved by using methods with adjustable sampling frequencies, such as the multiple-tau correlator ${ }^{26}$ and the order- $n$ algorithm. ${ }^{21,27}$ These methods can efficiently capture both slow and fast dynamics while keeping the computational requirements low. ${ }^{26,27}$ Thus, these advanced methods should be implemented in MD software.

LAMMPS $^{5}$ is an open-source MD code distributed under the terms of the GNU Public License. It is one of the most widely used MD packages because it features high degree of parallelization and size scalability as well as a modular structure, which promotes the development of new functionalities. ${ }^{28}$ LAMMPS is equipped with generic functionalities to compute transport coefficients based only on the Green-Kubo method. ${ }^{28}$ However, the Einstein approach combined with the order- $n$ algorithm, despite the advantage over the Green-Kubo method, has not been implemented.

We present a new plugin for LAMMPS for on-the-fly calculation of transport properties (OCTP) of fluids using the Einstein relations combined with the order- $n$ algorithm as presented in the work of Dubbeldam et al. ${ }^{27}$ The plugin can be used to compute the self- and Maxwell-Stefan (MS) diffusivity (based on the Onsager coefficients), the shear and bulk viscosity, and the thermal conductivity. The OCTP plugin has the following features: (1) Once invoked it computes all transport properties on-the-fly in a single simulation, so there is no need to store large trajectory files. (2) It uses the order- $n$ algorithm for the efficient sampling of MSD. (3) It has small CPU and storage requirements. (4) It is easy to setup and use because it follows the native format of LAMMPS input files. (5) All the data required for the calculation of MSDs are automatically written in restart files, so that long simulations can be performed in consecutive runs. The OCTP source code is available as open source and can be downloaded from https://github.com/ omoultosEthTuDelft/OCTP.

This application note is organized as follows. In section 2 , the methodology and the implementation of the code is briefly described. A case study using OCTP is provided in section 3, followed by the conclusion in the last section.

\section{METHODOLOGY AND CODE STRUCTURE}

The OCTP plugin is implemented in the LAMMPS ${ }^{5}$ version released on November 27, 2018, and consists of child-classes of the "compute" and "fix" parent-classes. Information on the source code structure of LAMMPS along with instructions for modifications and extensions can be found in the online manual. ${ }^{28}$ Consistent with the structure of LAMMPS input commands, ${ }^{28}$ arguments can be specified to adjust the parameters of the plugin. Such arguments include the choice of transport properties to be calculated, details of the order- $n$ algorithm (e.g., the number of blocks), and the names and the format of the output files. A detailed description of all available options of the OCTP plugin is presented in the Supporting Information.

When OCTP is invoked, the dynamical variables of the system are sampled using the relevant "compute" commands and stored in memory according to the order- $n$ algorithm. For the computation of self- and MS diffusion coefficients, the positions of atoms are sampled. For the bulk and shear viscosities and thermal conductivity, the components of the stress tensor and heat flux are sampled, respectively. These quantities are then integrated according to the Simpson's rule ${ }^{29}$ in the OCTP plugin and the results are stored in memory. It is important to note that the dynamical variables for the viscosity and thermal conductivity should be sampled rather frequently, in the range of $1-10$ timesteps. Less frequent sampling would yield unreliable viscosities and thermal conductivities, as the accuracy of numerical integration depends on the time intervals at which a property is sampled.

Besides the MS diffusivity, the Fick mutual diffusion coefficient can be computed from EMD simulations. ${ }^{30-32}$ These two diffusivities are related via the so-called thermodynamic factor, $\Gamma .^{33-35}$ One method for calculating $\Gamma$ from $M D$ is via Kirkwood-Buff coefficients, ${ }^{32,36-38}$ which require the computation of radial distribution functions (RDF) for distances larger than the cutoff radius, e.g. half the length of the simulation box. For a detailed description of this method the reader is referred to the work of Krüger et al. ${ }^{37,39}$ Although there is a generic command for computing RDFs in LAMMPS, it does not provide the RDF beyond the cutoff radius and finite-size effects are not considered. In our plugin, finite-size effects of RDFs are calculated according to the work of van der Vegt and co-workers. ${ }^{40,41}$ OCTP also computes RDFs up to $\sqrt{2} / 2$ of the box length. In the study by Theodorou and Suter ${ }^{42}$ it is shown that the calculation of RDFs can be extended up to $\sqrt{3} / 2$ of the box length. Close to this upper limit, the computed RDFs are prone to high statistical uncertainties. ${ }^{42}$ The van der Vegt correction $^{40,41}$ combined with the large-distance sampling of RDFs (i.e., beyond the cutoff radius) facilitates the computation of thermodynamic factors using the approach of Krüger and coworkers. $^{37,39}$ 
For each transport property, the OCTP plugin generates output files containing the correlation time and the corresponding MSD $\left((A(t)-A(0))^{2}\right)$. At time scales where the MSD becomes a linear function, the transport property (i.e., the coefficient of proportionality) is obtained by performing linear regression. The commands used in the LAMMPS input file as well as the output files generated by the OCTP plugin are explained in detail in the Supporting Information, along with a description of the Einstein relations used in the code. For more information on the calculation of transport properties using the Einstein relations, the reader is referred to the relevant textbooks, ${ }^{4,21,43}$ the review article by Zwanzig, ${ }^{19}$ and to other relevant articles on computing diffusivities, ${ }^{32,44-47}$ viscosities, $^{48-52}$ and thermal conductivities. ${ }^{53}$

\section{CASE STUDY}

To demonstrate the new plugin, MD simulations were performed to compute the self- and MS diffusion coefficient, shear and bulk viscosity, and thermal conductivity of a watermethanol mixture $\left(x_{\text {methanol }}=0.5\right)$. All simulations were performed in the microcanonical (NVE) ensemble at a corresponding temperature and pressure of $298 \mathrm{~K}$ and $1 \mathrm{~atm}$, respectively. The simulation details along with the corresponding LAMMPS files are provided in the Supporting Information.

In Figure 2, the MSD for the self-diffusion coefficient of methanol and water is shown in a $\log -\log$ plot. A slope of 1 is

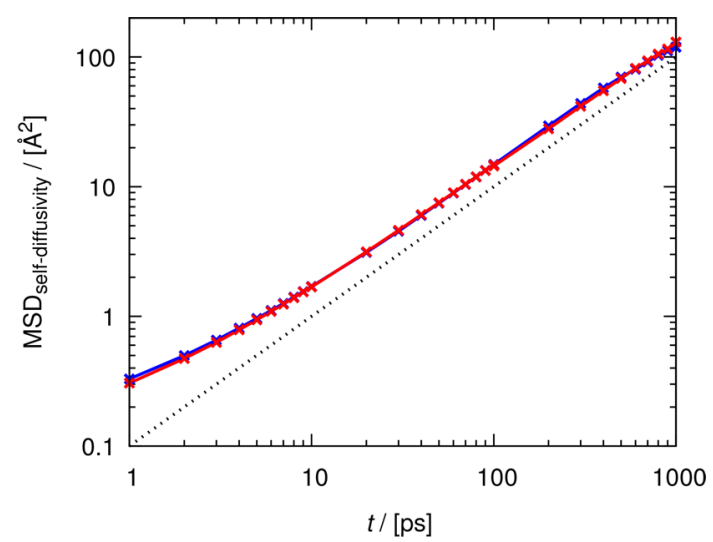

Figure 2. Computed MSD (right-hand side of eq 2) as a function of the correlation time (log-log plot) for water (blue) and methanol (red) in an equimolar mixture of water-methanol at $298 \mathrm{~K}$ and $1 \mathrm{bar}$. Solid lines are computed from the OCTP plugin, while crosses represent the MSDs obtained by postprocessing the trajectory files. Self-diffusivities can be calculated by performing linear regression at time scales where the slope of MSD in this plot is equal to 1 (dashed line). ${ }^{27}$ The MSD for the other transport properties are presented in Figure S5 of the Supporting Information. The simulation details along with the corresponding LAMMPS files are provided in the Supporting Information.

observed beyond $50 \mathrm{ps}$. Due to the smaller number of samples at large time scales and possible accumulation of noise, linear regression should be performed at the smallest correlation time for which MSD is a linear function of time ${ }^{54}$ (here between 10 and $100 \mathrm{ps}$ ). The MSD for the other transport properties are presented in Figure S5 of the Supporting Information. To validate that the new plugin is implemented correctly, the transport properties of the water-methanol mixture were computed on-the-fly with OCTP and then compared with the results from the conventional Green-Kubo method. As computed with OCTP the self-diffusivity of water and methanol is $1.50 \times 10^{-9}$ and $1.48 \times 10^{-9} \mathrm{~m}^{2} \mathrm{~s}^{-1}$, respectively, the MS mutual diffusivity is $2.2 \times 10^{-9} \mathrm{~m}^{2} \mathrm{~s}^{-1}$, the shear viscosity is 0.83 $\mathrm{cP}$, and the thermal conductivity is $0.39 \mathrm{~W} \mathrm{~m}^{-1} \mathrm{~K}^{-1}$. The results from the Green-Kubo method are the self-diffusivities of water and methanol at $1.61 \times 10^{-9}$ and $1.48 \times 10^{-9} \mathrm{~m}^{2} \mathrm{~s}^{-1}$, respectively, the MS mutual diffusivity is $2.1 \times 10^{-9} \mathrm{~m}^{2} \mathrm{~s}^{-1}$, the shear viscosity is $0.85 \mathrm{cP}$, and the thermal conductivity is $0.38 \mathrm{~W}$ $\mathrm{m}^{-1} \mathrm{~K}^{-1}$. The agreement between the two methods is very good (within approximately $5 \%$ ).

It is important to note that diffusion coefficients and thermal conductivities depend on the system size and should be corrected for obtaining the values in the thermodynamic limit. ${ }^{55-58}$ Especially close to the critical point, transport properties can show considerable finite-size effects. ${ }^{59}$ To that purpose, the OCTP plugin was used to investigate the finite-size effects of the self-diffusion coefficient, shear viscosity, bulk viscosity, and thermal conductivity of a Lennard-Jones fluid close to the critical point. The results are shown in the Supporting Information.

To demonstrate the computational efficiency and size scalability for the calculation of each transport property with the OCTP plugin, MD simulations were performed for six system sizes (i.e., 250, 500, 1000, 2000, 4000, and 8000 molecules) on a 32-core processing unit (Intel Xeon Processor E5-2697Av4@2.60 GHz).The results are presented in Figure 3

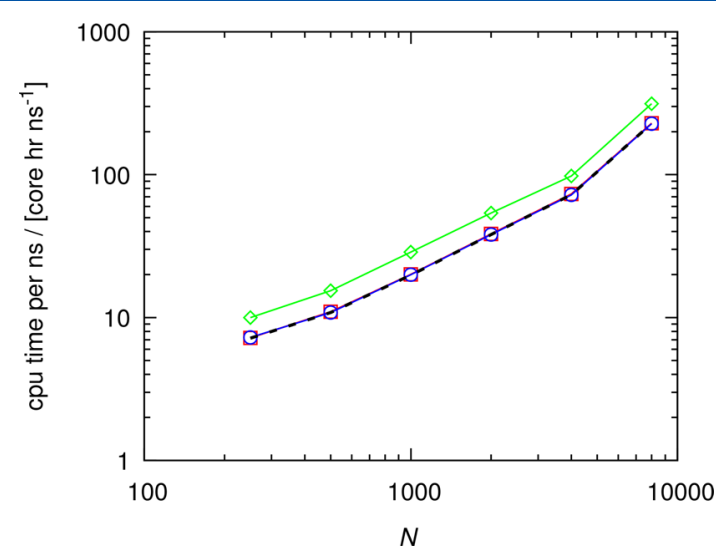

Figure 3. Computational requirements and size scalability of the OCTP plugin for simulations performing the calculation of diffusion coefficients (blue circles), viscosities (red squares), and thermal conductivity (green diamonds) for different system sizes of an equimolar binary mixture of water-methanol. The dashed line shows computational requirements for simulations without invoking OCTP. The total number of molecules ranges from 250 to 8000 molecules. $T=298 \mathrm{~K}$ and $P=1 \mathrm{~atm}$. All simulations were performed on a 32-core processor. Lines are intended only as guide to the eye.

for a water-methanol mixture $\left(x_{\text {methanol }}=0.5\right)$. Diffusion and viscosity calculations consume less than $2 \%$ of the total computational resources, while thermal conductivity calculation consumes approximately $45 \%$. The increased computational requirement for the calculation of thermal conductivity is justified from the intensive calculation of the per-atom properties needed, regardless of the sampling scheme implemented. As mentioned earlier, in the OCTP plugin transport coefficient calculations can be switched on or off individually. Therefore, the user can optimize the simulations by switching on only the properties needed. Based on these results, 
we can conclude that the new plugin is highly efficient for onthe-fly calculations.

\section{CONCLUSIONS}

A new plugin implemented in LAMMPS, called OCTP, is presented for on-the-fly computations of the self- and MaxwellStefan diffusivities, the bulk and shear viscosities, and the thermal conductivity of pure fluids and mixtures in equilibrium molecular dynamics. This is the first implementation in LAMMPS that uses the Einstein relations combined with the order- $n$ algorithm for the efficient sampling of dynamic variables. The OCTP plugin yields all transport coefficients in a single simulation, has low CPU and storage requirements and is easy to use since it follows the native LAMMPS input file format. OCTP also features a tool for calculating the radial distribution function of the fluid beyond the cutoff radius, while taking into account system size effects. This precise RDF calculation can be used to calculate the thermodynamic factor, $\Gamma$, and thus the Fick mutual diffusivity. As a case study, the transport properties for an equimolar mixture of water-methanol were computed at $298 \mathrm{~K}$ and 1 bar. It was found that the computational requirements of OCTP are low and thus, the new plugin can be used for efficient on-the-fly calculations of transport properties.

\section{ASSOCIATED CONTENT}

\section{(S Supporting Information}

The Supporting Information is available free of charge on the ACS Publications website at DOI: 10.1021/acs.jcim.8b00939.

Description of the Einstein relations used in the OCTP plugin, the installation instructions of OCTP in LAMMPS, the description of the OCTP commands for the LAMMPS input file, sample output files obtained from the OCTP, finite-size effects of transport properties close to the critical point for a Lennard-Jones fluid, and the details for simulating a water-methanol mixture (PDF)

Sample input files for LAMMPS for the watersystem used in the study (ZIP)

\section{AUTHOR INFORMATION}

\section{Corresponding Author}

*E-mail: o.moultos@tudelft.nl.

\section{ORCID 8}

Seyed Hossein Jamali: 0000-0002-4198-0901

Ludger Wolff: 0000-0001-7171-7947

Tim M. Becker: 0000-0002-6601-4320

Mahinder Ramdin: 0000-0002-8476-7035

Remco Hartkamp: 0000-0001-8746-8244

André Bardow: 0000-0002-3831-0691

Thijs J. H. Vlugt: 0000-0003-3059-8712

Othonas A. Moultos: 0000-0001-7477-9684

\section{Notes}

The authors declare no competing financial interest.

The OCTP plugin can be downloaded as open-source from https://github.com/omoultosEthTuDelft/OCTP.

\section{ACKNOWLEDGMENTS}

This work was sponsored by NWO Exacte Wetenschappen (Physical Sciences) for the use of supercomputer facilities, with financial support from the Nederlandse Organisatie voor Wetenschappelijk Onderzoek (Netherlands Organization for
Scientific Research, NWO). T.J.H.V. acknowledges NWO-CW (Chemical Sciences) for a VICI grant.

\section{REFERENCES}

(1) Bird, R. B. Transport Phenomena, 2nd ed.; John Wiley \& Sons: New York, 2007.

(2) Poling, B. E.; Prausnitz, J. M.; O'Connel, J. P. The Properties of Gases and Liquids, 5th ed.; McGraw-Hill: Singapore, 2001.

(3) Economou, I.; Krokidas, P.; Michalis, V.; Moultos, O.; Tsimpanogiannis, I.; Vergadou, N. Water-Food-Energy Nexus Process. Technol. Challenges; CRC Press: Boca Raton, 2017; Chapter 13, pp 633-660.

(4) Allen, M. P.; Tildesley, D. J. Computer Simulation of Liquids, 2nd ed.; Oxford University Press: Croydon, 2017.

(5) Plimpton, S. Fast Parallel Algorithms for Short-Range Molecular Dynamics. J. Comput. Phys. 1995, 117, 1-19.

(6) Berendsen, H.; van der Spoel, D.; van Drunen, R. GROMACS: A message-passing parallel molecular dynamics implementation. Comput. Phys. Commun. 1995, 91, 43-56.

(7) Phillips, J. C.; Braun, R.; Wang, W.; Gumbart, J.; Tajkhorshid, E.; Villa, E.; Chipot, C.; Skeel, R. D.; Kalé, L.; Schulten, K. Scalable Molecular Dynamics with NAMD. J. Comput. Chem. 2005, 26, 17811802.

(8) Jamali, S. H.; Ramdin, M.; Becker, T. M.; Rinwa, S. K.; Buijs, W.; Vlugt, T. J. H. Thermodynamic and Transport Properties of CrownEthers: Force Field Development and Molecular Simulations. J. Phys. Chem. B 2017, 121, 8367-8376.

(9) Budhathoki, S.; Shah, J. K.; Maginn, E. J. Molecular Simulation Study of the Performance of Supported Ionic Liquid Phase Materials for the Separation of Carbon Dioxide from Methane and Hydrogen. Ind. Eng. Chem. Res. 2017, 56, 6775-6784.

(10) Moultos, O. A.; Tsimpanogiannis, I. N.; Panagiotopoulos, A. Z.; Economou, I. G. Atomistic Molecular Dynamics Simulations of $\mathrm{CO}_{2}$ Diffusivity in $\mathrm{H}_{2} \mathrm{O}$ for a Wide Range of Temperatures and Pressures. J. Phys. Chem. B 2014, 118, 5532-5541.

(11) Moultos, O. A.; Tsimpanogiannis, I. N.; Panagiotopoulos, A. Z.; Economou, I. G. Self-Diffusion Coefficients of the Binary $\left(\mathrm{H}_{2} \mathrm{O}+\mathrm{CO}_{2}\right)$ Mixture at High Temperatures and Pressures. J. Chem. Thermodyn. 2016, 93, 424-429.

(12) Otani, A.; Zhang, Y.; Matsuki, T.; Kamio, E.; Matsuyama, H.; Maginn, E. J. Molecular Design of High $\mathrm{CO}_{2}$ Reactivity and Low Viscosity Ionic Liquids for $\mathrm{CO}_{2}$ Separative Facilitated Transport Membranes. Ind. Eng. Chem. Res. 2016, 55, 2821-2830.

(13) Jiang, H.; Mester, Z.; Moultos, O. A.; Economou, I. G.; Panagiotopoulos, A. Z. Thermodynamic and Transport Properties of $\mathrm{H}_{2} \mathrm{O}+\mathrm{NaCl}$ from Polarizable Force Fields. J. Chem. Theory Comput. 2015, 11, 3802-3810.

(14) Jamali, S. H.; Vlugt, T. J. H.; Lin, L.-C. Atomistic Understanding of Zeolite Nanosheets for Water Desalination. J. Phys. Chem. C 2017, $121,11273-11280$

(15) Lin, L.-C.; Grossman, J. C. Atomistic understandings of reduced graphene oxide as an ultrathin-film nanoporous membrane for separations. Nat. Commun. 2015, 6, 8335.

(16) Wolff, L.; Jamali, S. H.; Becker, T. M.; Moultos, O. A.; Vlugt, T. J. H.; Bardow, A. Prediction of Composition-Dependent Self-Diffusion Coefficients in Binary Liquid Mixtures: The Missing Link for DarkenBased Models. Ind. Eng. Chem. Res. 2018, 57, 14784-14794.

(17) Moggridge, G. Prediction of the mutual diffusivity in binary nonideal liquid mixtures from the tracer diffusion coefficients. Chem. Eng. Sci. 2012, 71, 226-238.

(18) Tenney, C. M.; Maginn, E. J. Limitations and Recommendations for the Calculation of Shear Viscosity using Reverse Nonequilibrium Molecular Dynamics. J. Chem. Phys. 2010, 132, 014103.

(19) Zwanzig, R. Time-Correlation Functions and Transport Coefficients in Statistical Mechanics. Annu. Rev. Phys. Chem. 1965, $16,67-102$.

(20) Evans, D. J.; Morriss, G. Statistical Mechanics of Nonequilibrium Liquids, 2nd ed.; Cambridge University Press: Cambridge, 2008. 
(21) Frenkel, D.; Smit, B. Understanding Molecular Simulation: From Algorithms to Applications, 2nd ed.; Academic Press: London, 2002.

(22) Hagen, M. H. J.; Lowe, C. P.; Frenkel, D. Long Time Tails in Stress Correlation Functions. 25 Years Non-Equil. Stat. Mechan. 2005, $445,240-249$.

(23) van der Hoef, M. A.; Frenkel, D. Long-time Tails of the Velocity Autocorrelation Function in Two- and Three-dimensional Lattice-gas Cellular Automata: A Test of Mode-Coupling Theory. Phys. Rev. A: At., Mol., Opt. Phys. 1990, 41, 4277-4284.

(24) Zhang, Y.; Otani, A.; Maginn, E. J. Reliable Viscosity Calculation from Equilibrium Molecular Dynamics Simulations: A Time Decomposition Method. J. Chem. Theory Comput. 2015, 11, 3537-3546.

(25) Maginn, E. J.; Messerly, R. A.; Carlson, D. J.; Roe, D. R.; Elliott, J. R. Best Practices for Computing Transport Properties 1. Self-Diffusivity and Viscosity from Equilibrium Molecular Dynamics [Article v1.0]. Living J. Comput. Mol. Sci. 2019, 1, 1-20.

(26) Ramírez, J.; Sukumaran, S. K.; Vorselaars, B.; Likhtman, A. E. Efficient on the Fly Calculation of Time Correlation Functions in Computer Simulations. J. Chem. Phys. 2010, 133, 154103.

(27) Dubbeldam, D.; Ford, D. C.; Ellis, D. E.; Snurr, R. Q. A New Perspective on the Order-n Algorithm for Computing Correlation Functions. Mol. Simul. 2009, 35, 1084-1097.

(28) Sandia National Laboratories. LAMMPS Documentation. https://lammps.sandia.gov/doc/Manual.html.

(29) Press, W. H.; Teukolsky, S. A.; Vetterling, W. T.; Flannery, B. P. Numerical Recipes: The Art of Scientific Computing, 3rd ed.; Cambridge University Press: Hong Kong, 2007.

(30) Nichols, J. W.; Wheeler, D. R. Fourier Correlation Method for Simulating Mutual Diffusion Coefficients in Condensed Systems at Equilibrium. Ind. Eng. Chem. Res. 2015, 54, 12156-12164.

(31) Liu, X.; Martín-Calvo, A.; McGarrity, E.; Schnell, S. K.; Calero, S.; Simon, J.-M.; Bedeaux, D.; Kjelstrup, S.; Bardow, A.; Vlugt, T. J. H. Fick Diffusion Coefficients in Ternary Liquid Systems from Equilibrium Molecular Dynamics Simulations. Ind. Eng. Chem. Res. 2012, 51, 10247-10258.

(32) Liu, X.; Schnell, S. K.; Simon, J.-M.; Krüger, P.; Bedeaux, D.; Kjelstrup, S.; Bardow, A.; Vlugt, T. J. H. Diffusion Coefficients from Molecular Dynamics Simulations in Binary and Ternary Mixtures. Int. J. Thermophys. 2013, 34, 1169-1196.

(33) Krishna, R.; van Baten, J. M. MD Simulations of Diffusivities in Methanol-n-hexane Mixtures Near the Liquid-liquid Phase Splitting Region. Chem. Eng. Technol. 2006, 29, 516-519.

(34) Krishna, R.; van Baten, J. M. Describing Diffusion in Fluid Mixtures at Elevated Pressures by Combining the Maxwell-Stefan Formulation with an Equation of State. Chem. Eng. Sci. 2016, 153, 174187.

(35) Krishna, R.; Wesselingh, J. The Maxwell-Stefan Approach to Mass Transfer. Chem. Eng. Sci. 1997, 52, 861-911.

(36) Ben-Naim, A. Molecular Theory of Solutions; Oxford University Press: Oxford, 2006.

(37) Krüger, P.; Vlugt, T. J. H. Size and Shape Dependence of Finitevolume Kirkwood-Buff Integrals. Phys. Rev. E: Stat. Phys., Plasmas, Fluids, Relat. Interdiscip. Top. 2018, 97, 051301.

(38) Dawass, N.; Krüger, P.; Schnell, S. K.; Simon, J.-M.; Vlugt, T. J. H. Kirkwood-Buff Integrals from Molecular Simulation. Fluid Phase Equilib. 2019, 486, 21-36.

(39) Krüger, P.; Schnell, S. K.; Bedeaux, D.; Kjelstrup, S.; Vlugt, T. J. H.; Simon, J.-M. Kirkwood-Buff Integrals for Finite Volumes. J. Phys. Chem. Lett. 2013, 4, 235-238.

(40) Ganguly, P.; van der Vegt, N. F. A. Convergence of Sampling Kirkwood-Buff Integrals of Aqueous Solutions with Molecular Dynamics Simulations. J. Chem. Theory Comput. 2013, 9, 1347-1355.

(41) Milzetti, J.; Nayar, D.; van der Vegt, N. F. A. Convergence of Kirkwood-Buff Integrals of Ideal and Nonideal Aqueous Solutions Using Molecular Dynamics Simulations. J. Phys. Chem. B 2018, 122, $5515-5526$

(42) Theodorou, D. N.; Suter, U. W. Geometrical Considerations in Model Systems with Periodic Boundaries. J. Chem. Phys. 1985, 82, 955-966.
(43) Hansen, J.-P.; McDonald, I. R. Theory of Simple Liquids, 3rd ed.; Academic Press: Amsterdam, 2006.

(44) Meier, K.; Laesecke, A.; Kabelac, S. Transport coefficients of the Lennard-Jones model fluid. II Self-diffusion. J. Chem. Phys. 2004, 121, 9526-9535.

(45) Pranami, G.; Lamm, M. H. Estimating Error in Diffusion Coefficients Derived from Molecular Dynamics Simulations. J. Chem. Theory Comput. 2015, 11, 4586-4592.

(46) Moultos, O. A.; Tsimpanogiannis, I. N.; Panagiotopoulos, A. Z.; Trusler, J. P. M.; Economou, I. G. Atomistic Molecular Dynamics Simulations of Carbon Dioxide Diffusivity in n-Hexane, n-Decane, nHexadecane, Cyclohexane, and Squalane. J. Phys. Chem. B 2016, 120, 12890-12900.

(47) Michalis, V. K.; Moultos, O. A.; Tsimpanogiannis, I. N.; Economou, I. G. Molecular Dynamics Simulations of the Diffusion Coefficients of Light n-Alkanes in Water over a Wide Range of Temperature and Pressure. Fluid Phase Equilib. 2016, 407, 236-242.

(48) Mondello, M.; Grest, G. S. Viscosity Calculations of n-Alkanes by Equilibrium Molecular Dynamics. J. Chem. Phys. 1997, 106, 9327.

(49) Borodin, O.; Smith, G. D.; Kim, H. Viscosity of a Room Temperature Ionic Liquid: Predictions from Nonequilibrium and Equilibrium Molecular Dynamics Simulations. J. Phys. Chem. B 2009, $113,4771-4774$.

(50) Meier, K.; Laesecke, A.; Kabelac, S. Transport coefficients of the Lennard-Jones model fluid. I. Viscosity. J. Chem. Phys. 2004, 121, 3671-3687.

(51) Meier, K.; Laesecke, A.; Kabelac, S. Transport coefficients of the Lennard-Jones model fluid. III. Bulk viscosity. J. Chem. Phys. 2005, 122, 014513 .

(52) Jamali, S. H.; Hartkamp, R.; Bardas, C.; Söhl, J.; Vlugt, T. J. H.; Moultos, O. A. Shear Viscosity Computed from the Finite-Size Effects of Self-Diffusivity in Equilibrium Molecular Dynamics. J. Chem. Theory Comput. 2018, 14, 5959-5968.

(53) Kinaci, A.; Haskins, J. B.; Cagin, T. On calculation of thermal conductivity from Einstein relation in equilibrium molecular dynamics. J. Chem. Phys. 2012, 137, 014106.

(54) Chen, T.; Smit, B.; Bell, A. T. Are Pressure Fluctuation-based Equilibrium Methods Really Worse than Nonequilibrium Methods for Calculating Viscosities? J. Chem. Phys. 2009, 131, 246101.

(55) Yeh, I.-C.; Hummer, G. System-Size Dependence of Diffusion Coefficients and Viscosities from Molecular Dynamics Simulations with Periodic Boundary Conditions. J. Phys. Chem. B 2004, 108, 15873-15879.

(56) Sellan, D. P.; Landry, E. S.; Turney, J. E.; McGaughey, A. J. H.; Amon, C. H. Size Effects in Molecular Dynamics Thermal Conductivity Predictions. Phys. Rev. B: Condens. Matter Mater. Phys. 2010, 81, 214305.

(57) Moultos, O. A.; Zhang, Y.; Tsimpanogiannis, I. N.; Economou, I. G.; Maginn, E. J. System-Size Corrections for Self-Diffusion Coefficients Calculated from Molecular Dynamics Simulations: The Case of $\mathrm{CO}_{2}$, n-alkanes, and Poly(Ethylene Glycol) Dimethyl Ethers. J. Chem. Phys. 2016, 145, 074109.

(58) Jamali, S. H.; Wolff, L.; Becker, T. M.; Bardow, A.; Vlugt, T. J. H.; Moultos, O. A. Finite-Size Effects of Binary Mutual Diffusion Coefficients from Molecular Dynamics. J. Chem. Theory Comput. 2018, 14, 2667-2677.

(59) Das, S. K.; Fisher, M. E.; Sengers, J. V.; Horbach, J.; Binder, K. Critical Dynamics in a Binary Fluid: Simulations and Finite-Size Scaling. Phys. Rev. Lett. 2006, 97, 025702.

\section{NOTE ADDED AFTER ASAP PUBLICATION}

This paper was published ASAP on February 21, 2019, missing one of its Supporting Information files. The corrected version was reposted on March 21, 2019. 